\title{
Correction to: Patients and surgeons provide endorsement of core domains for total joint replacement clinical trials
}

Anh Hoang', Susan M. Goodman', Iris Y. Navarro-Millán', Lisa A. Mandl', Mark P. Figgie², Mathias P. Bostrom², Douglas E. Padgett ${ }^{2}$, Peter K. Sculco ${ }^{2}$, Alexander S. McLawhorn ${ }^{2}$ and Jasvinder A. Singh $3,4,5,6^{*}$

\section{Correction}

Unfortunately, after publication of this article [1], it was noticed that Table 2 was not correctly formatted during the production process. The corrected Table 2 can be seen below and the original article has been updated to match.

Table 2 Domain ratings between patients and surgeons

\begin{tabular}{|c|c|c|c|c|}
\hline & $\begin{array}{l}\text { Overall } \\
(N=1316)\end{array}$ & $\begin{array}{l}\text { Patients } \\
(n=1295)\end{array}$ & $\begin{array}{l}\text { Surgeons } \\
(n=21)\end{array}$ & $p$ Value \\
\hline \multicolumn{5}{|l|}{ Core domains } \\
\hline Joint pain & $9(8-9)$ & $9(8-9)$ & $9(7-9)$ & 0.75 \\
\hline Function or functional ability & $9(8-9)$ & $9(8-9)$ & $8(7-9)$ & 0.01 \\
\hline Patient satisfaction & $9(8-9)$ & $9(8-9)$ & $8(8-9)$ & 0.02 \\
\hline Revision surgery & $8(5-9)$ & $8(5-9)$ & $8(7-8)$ & 0.41 \\
\hline Adverse events & $8(7-9)$ & $8(7-9)$ & $7(6-9)$ & 0.23 \\
\hline Death & $9(6-9)$ & $9(6-9)$ & $9(7-9)$ & 0.47 \\
\hline Additional domains for consideration & $\begin{array}{l}\text { Overall } \\
(N=1316)\end{array}$ & $\begin{array}{l}\text { Patients } \\
(n=1295)\end{array}$ & $\begin{array}{l}\text { Surgeons } \\
(n=21)\end{array}$ & $p$ Value \\
\hline Cost & $7(5-8)$ & $7(5-8)$ & $6(5-6)$ & 0.01 \\
\hline Patient participation in work and social activities & $8(6-9)$ & $8(6-9)$ & $8(6-8)$ & 0.26 \\
\hline
\end{tabular}

Each domain was rated on a 1-9 scale, with 1-3 indicating limited or no importance for patients, 4-6 being important but not critical, and 7-9 being critical Bold text signifies results that were statistically significant

\begin{abstract}
Author details
${ }^{1}$ Division of Rheumatology, Hospital for Special Surgery, New York, NY, USA ${ }^{2}$ Department of Orthopedic Surgery, Hospital for Special Surgery, New York, NY, USA. ${ }^{3}$ Medicine Service, VA Medical Center, 510 20th Street South, Faculty Office Tower 805B, Birmingham, AL, USA. ${ }^{4}$ Department of Medicine, School of Medicine, University of Alabama at Birmingham, 1720 Second Avenue South, Birmingham, AL 35294-0022, USA. ${ }^{5}$ Division of Epidemiology, School of Public Health, University of Alabama at Birmingham, 1720 Second Avenue South, Birmingham, AL 35294-0022, USA. ' University of Alabama at Birmingham, Faculty Office Tower 805B, 510 20th Street South, Birmingham AL 35294, USA.

\footnotetext{
*Correspondence: jasvinder.md@gmail.com

${ }^{3}$ Medicine Service, VA Medical Center, 510 20th Street South, Faculty Office Tower 805B, Birmingham, AL, USA

${ }^{4}$ Department of Medicine, School of Medicine, University of Alabama at

Birmingham, 1720 Second Avenue South, Birmingham, AL 35294-0022, USA
}

Received: 2 January 2018 Accepted: 2 January 2018

Published online: 14 February 2018

\section{Reference}

1. Hoang A, Goodman SM, Navarro-Millán IY, Mandl LA, Figgie MP, Bostrom $M P$, et al. Patients and surgeons provide endorsement of core domains for total joint replacement clinical trials. Arthritis Res Ther. 2017;19:267. https:// doi.org/10.1186/s13075-017-1476-9. 